J. Lake Sci.(湖泊科学), 2008, 20(3): 375-379

http://www.jlakes.org. E-mail: jlakes@niglas.ac.cn

(C)2008 by Journal of Lake Sciences

\title{
太湖水体的后向散射概率”
}

\author{
马荣华 ${ }^{1,2}$, 宋庆君 ${ }^{3}$, 李国砚 ${ }^{1}$, 潘德炉 ${ }^{2}$ \\ (1: 中国科学院南京地理与湖泊研究所, 南京 210008) \\ (2: 卫星海洋环境动力学国家重点实验室, 国家海洋局第二海洋研究所, 杭州 310012) \\ (3: 国家卫星海洋应用中心, 北京 100081)
}

摘 要: 假设 2004 年 10 月太湖水体的吸收系数和遥感反射比的测量误差为 0 , 利用生物光学模型, 通过优化算法, 在光学深 水区模拟获得水体的后向散射系数，然后通过后向散射概率和颗粒物后向散射系数之间的定量关系，获得水体中可能真实的 颗粒物后向散射概率. 结果表明，太湖水体的颗粒物后向散射概率不是一个定值，可以表示为波长的二次函数，在 $442 、 488 、$ $532 、 589 、 676$ 和 $852 \mathrm{~nm}$ 处分别为 $0.017 、 0.017 、 0.027 、 0.033 、 0.054$ 和 0.094 , 均值为 0.041 , 标准偏差为 0.030 .

关键词: 后向散射概率; 后向散射系数; 颗粒物质; 太湖

\section{Estimation of backscattering probability of Lake Taihu waters}

\author{
MA Ronghua ${ }^{1,2}$, SONG Qingjun ${ }^{3}$, LI Guoyan ${ }^{1} \&$ PAN Delu ${ }^{2}$ \\ (1: Nanjing Institute of Geography and Limnology, Chinese Academy of Sciences, Nanjing 210008, P.R.China) \\ (2: State Key Laboratory of Satellite Ocean Environment Dynamics, Second Institute of Oceanography, State Oceanic Administration, \\ Hangzhou 310012, P.R.China) \\ (3: National Satellite Ocean Application Service, Beijing 100081, P.R.China)
}

\begin{abstract}
We assume that the measurement errors of absorption coefficient and remote sensing reflectance are zero in October 2004, Lake Taihu. Then we can employ the optimization algorithm to simulate the backscattering coefficient by the most appropriate bio-optical model especially in the optically deep waters, where the bottom has no influence on remote sensing reflectance. Finally, the possibly real backscattering probability of suspended particulate matter may be approximately calculated by the quantitative relationship between the tested backscattering probabilities and their corresponding backscattering coefficients of suspended particulate matter. The results show that the backscattering probability of suspended particulate matter is not a constant and can be expressed as a quadratic function of wavelength, and the values are 0.017, 0.017, 0.027, 0.033, 0.054 and 0.094 at 442, 488, 532, 589, 676 and $852 \mathrm{~nm}$, respectively; with a mean of $0.041 \pm 0.030$.
\end{abstract}

Keywords: Backscattering probability; backscattering coefficient; suspended particulate matter; Lake Taihu

后向散射概率(backscattering probability, $\tilde{b}_{\mathrm{b}}$ )是后向散射与总散射的比值(the ratio of the scattering in the backward hemisphere to the total scattering occurring in all directions, i.e. $\left.b_{\mathrm{b}} / b\right)$, 也有学者定义为颗粒物 后向散射与颗粒物散射的比值(the ratio between the backscattering coefficient and the scattering coefficient of the particles, i.e. $b_{\mathrm{bp}} / b_{\mathrm{p}}$ ). 在后向散射系数的现场测量过程中, 为补偿光衰减而引起的数值低估, 一般都 要使用后向散射概率进行 sigma 校正 ${ }^{[1]}$, 校正时一般假设后向散射概率为已知量, 因此后向散射概率影响 着后向散射系数的现场测量精度. 大洋开阔水体中, 后向散射概率 $b_{\mathrm{b}} / b$ 基本稳定, HS-6(HydroScat-6 spectral backscattering sensor)默认为 $0.015^{[2]}, \mathrm{Kirk}^{[3]}$ 认为对许多沿岸和中混浊水体而言, 0.019 较为适合; Whitlock 等 ${ }^{[4]}$ 认为混浊河水的后向散射概率大约为 $0.059 ;$ Aas 等 ${ }^{[5]}$ 的研究表明, $b_{\mathrm{bp}} / b_{\mathrm{p}}$ 与悬浮颗粒大小有

* 国家自然科学基金(40671138)、浙江省自然科学基金重点项目(Z507024)和江苏省自然科学基金(BK2007261)联合资助. 2007-04-12 收稿; 2007-11-06 收修改稿. 马荣华, 男, 1972 年生, 博士, 副研究员; E-mail: rhma@niglas.ac.cn. 

关, 颗粒越小, $b_{\mathrm{bp}} / b_{\mathrm{p}}$ 越大, 一般在 0.017 到 0.029 之间变化, 上限为 0.5 . 可见, 不同的水体, 后向散射概率 有着较大的差异. 本文试图通过现场测量, 结合优化算法, 给出太湖水体较为准确的后向散射概率, 从 而为准确的后向散射系数获取以及高精度太湖水质遥感监测提供基础.

\section{1 方法}

在 2004 年 10 月后向散射系数的现场测量与校正过程 ${ }^{\left[{ }^{1]}\right.}$ 中, 首先使用了 HS-6 默认的后向散射概率 0.015 , 然后又试着使用了 $0.019 、 0.030$ 和 0.050 (称为试用后向散射概率, the tested backscattering probability, $\tilde{b}_{\mathrm{bt}}$ ), 得到了四组后向散射系数数据(backscattering coefficient, $b_{\mathrm{b}}$ ), 称为测量的后向散射系数 (the measured backscattering coefficient, $b_{\mathrm{bm}}$ ), 有

$$
b_{\mathrm{bm}}=b_{\mathrm{bw}}+b_{\mathrm{bpm}}
$$

式中, $b_{\mathrm{bw}}$ 是纯水的后向散射系数, 为已知量 ${ }^{[6]}, b_{\mathrm{bpm}}$ 是与 $b_{\mathrm{bm}}$ 对应的颗粒物的后向散射系数, 称为测量的 颗粒物后向散射系数(the measured backscattering coefficient of suspended particulate matter), 记波长 $\lambda_{i}$ 处的 均值为 $\operatorname{mean}\left(b_{\mathrm{bpm}_{i}}\right)$, 有

$$
\operatorname{mean}\left(b_{\mathrm{bpm}_{i}}\right)=\sum_{j=1}^{N} b_{\mathrm{bpm}_{j}}\left(\lambda_{i}\right) / N
$$

式中, $i$ 表示波长序号 $(1 、 2 、 3 、 4 、 5$ 和 6 分别表示 $442 、 488 、 532 、 589 、 676$ 和 $852 \mathrm{~nm}) ; j$ 是样本号, $N$ 是样本总数. 一般情况下, 在不同波长处, 试用后向散射概率 $\tilde{b}_{\mathrm{bt}}$ 与其对应的颗粒物后向散射系数之间存 在可定量的经验关系. 理想情况下，颗粒物后向散射系数 (backscattering coefficient of suspended particulate matter, $\left.b_{\mathrm{bp}}\right)$ 可以表达为波长的幂函数, 有 ${ }^{[6-9]}$

$$
b_{\mathrm{bp}}(\lambda)=b_{\mathrm{bp}}\left(\lambda_{0}\right)\left(\frac{\lambda_{0}}{\lambda}\right)^{n}
$$

式中, $n$ 是波形指数, $\lambda_{0}$ 是参考波长, 一般取 $550 \mathrm{~nm}^{[10]}$; 为了与 HS-6 的波段 $(442 、 488 、 532 、 589 、 676$ 和 $852 \mathrm{~nm}$ ) 相对应, 本文取 $532 \mathrm{~nm}$. 对于太湖水体而言, 最适合的生物光学模型有 ${ }^{[1]}$ :

$$
R_{\mathrm{rs}}=\frac{f}{Q} \frac{b_{\mathrm{b}}}{a+b_{\mathrm{b}}}=f^{\prime} \frac{b_{\mathrm{b}}}{a+b_{\mathrm{b}}}
$$

式中, $f=0.975-0.629 \mu_{0}^{[11]}, \mu_{0}$ 是刚好处于水面以下的太阳折射角的平均余弦(the mean cosine of the angles the photons make with vertical just below the surface), $Q$ 是水下光场分布函数, 表达水下光场的异质性特征, 可以定义为恰在水面下的向上辐照度(upwelling irradiance)与向下辐亮度(downwelling radiance)的比值; $R_{\mathrm{rs}}$ 为遥感反射比(remote sensing reflectance), $a$ 为总吸收系数, 是浮游植物色素吸收、非藻类颗粒物吸收、 CDOM(Colored Dissolved Organic Matter)吸收以及纯水吸收的总和, 上述光学量的测量过程和步骤详见 文献 ${ }^{[1]}$ 和 ${ }^{[12]}$. 把(1)和(3)式代人(4)式, 有:

$$
R_{\mathrm{rs}}(\lambda)=f^{\prime} \frac{b_{\mathrm{bw}}(\lambda)+b_{\mathrm{bp}}(532)\left(\frac{532}{\lambda}\right)^{n}}{a(\lambda)+b_{\mathrm{bw}}(\lambda)+b_{\mathrm{bp}}(532)\left(\frac{532}{\lambda}\right)^{n}}
$$

假设吸收系数 $a(\lambda)$ 和遥感反射比 $R_{\mathrm{rs}}(\lambda)$ 是真实的, 无测量误差; 另外假设 $R_{\mathrm{rs}}(\lambda)$ 全部来自水体, 不受水 底的影响, 则式(5)中的 3 个未知量 $\left(f^{\prime} 、 b_{\mathrm{bp}}(532)\right.$ 和 $\left.n\right)$ 可以在满足最小二乘条件下通过优化算法来获取:

$$
\min \sum_{i=1}^{m}\left(R_{\mathrm{rsm}}\left(\lambda_{i}\right)-R_{\mathrm{rss}}\left(\lambda_{i}\right)\right)^{2}
$$

式中, $R_{\mathrm{rsm}}$ 和 $R_{\mathrm{rss}}$ 分别表示测量的和模拟的遥感反射比, $m$ 是波段数.

在太湖的光学深水区, 遥感反射比不受湖底的影响, 使用上述优化算法, 得到模拟的 $b_{\mathrm{bp}}(532)$, 进而 获得整个波长范围内的颗粒物后向散射系数, 称为模拟的颗粒物后向散射系数 (the simulated $b_{\mathrm{bp}}, b_{\mathrm{bps}}$ ). 假 设 $b_{\mathrm{bps}}$ 为真值, 通过已经建立的试用后向散射概率 $\tilde{b}_{\mathrm{bt}}$ 与其对应的颗粒物后向散射系数之间的经验关系, 
即可获得可能真实的后向散射概率(the possibly real backscattering probability, $\tilde{b}_{\mathrm{bt}}$ ).

\section{2 结果}

为了与模拟结果相对应, 分析仅限于光学深水区(遥感反射比不受底质的影响, 包括高混浊水体区和 低混浊水体区)，共 41 个样点 ${ }^{[1]}$. 现场测量结果表明，随着试用后向散射概率 $\tilde{b}_{\mathrm{bt}}$ 从 0.015 到 0.050 的不断 增加，在同一波长处，对应的颗粒物后向散射系数不断减小; 在红外近红外波长范围内，高混浊水体以及 部分中混浊水体颗粒物后向散射系数随波长而不断增加, 这与理论上或模拟结果不断减小的趋势相背, 表明不恰当的后向散射系数校正量. 在 6 个波长处(442、488、532、589、676、852nm), 试用后向散射概 率 $\tilde{b}_{\mathrm{bt}}$ 与对应测量的后向散射系数之间存在明显的幂函数关系(图 1), 有:

$$
\begin{array}{ll}
\tilde{b}_{\mathrm{bt}}=0.0215 \times \operatorname{mean}\left(b_{\mathrm{bpm}}\right)^{-1.8075}, & R^{2}=0.9737, \quad \lambda=442 \mathrm{~nm} \\
\tilde{b}_{\mathrm{bt}}=0.0139 \times \operatorname{mean}\left(b_{\mathrm{bpm}}\right)^{-1.7584}, & R^{2}=0.9737, \quad \lambda=488 \mathrm{~nm} \\
\tilde{b}_{\mathrm{bt}}=0.0172 \times \operatorname{mean}\left(b_{\mathrm{bpm}}\right)^{-1.4743}, & R^{2}=0.9735, \quad \lambda=532 \mathrm{~nm} \\
\tilde{b}_{\mathrm{bt}}=0.0153 \times \operatorname{mean}\left(b_{\mathrm{bpm}}\right)^{-1.4317}, & R^{2}=0.9734, \quad \lambda=589 \mathrm{~nm} \\
\tilde{b}_{\mathrm{bt}}=0.0170 \times \operatorname{mean}\left(b_{\mathrm{bpm}}\right)^{-1.3596}, & R^{2}=0.9727, \quad \lambda=676 \mathrm{~nm} \\
\tilde{b}_{\mathrm{bt}}=0.0149 \times \operatorname{mean}\left(b_{\mathrm{bpm}}\right)^{-1.3472}, & R^{2}=0.9720, \quad \lambda=852 \mathrm{~nm}
\end{array}
$$
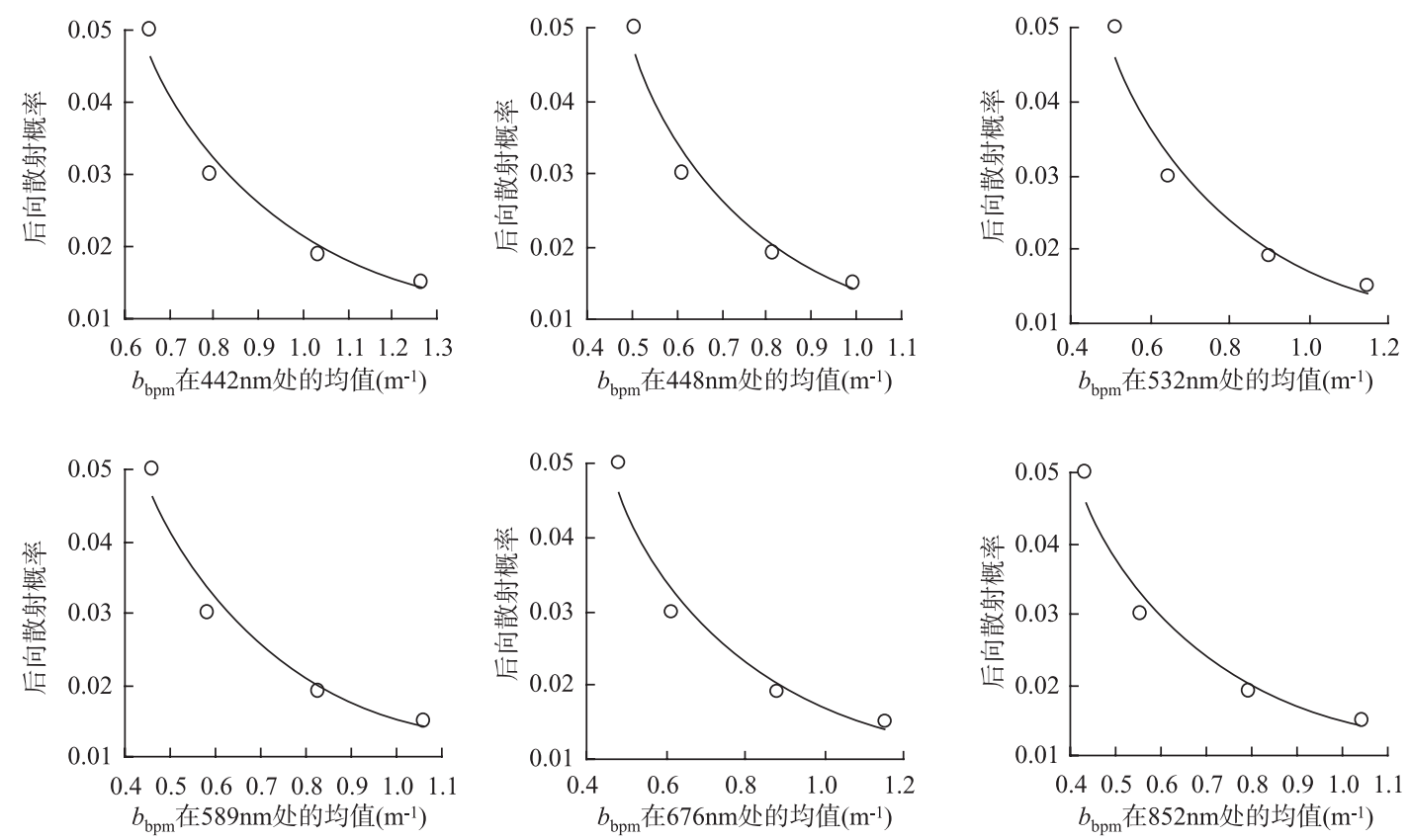

图 1442、488、532、589、676 和 $852 \mathrm{~nm}$ 处测量的悬浮颗粒后向散射系数与其对应的试用后向散射概率 之间的函数关系

Fig.1 Relationships between backscattering probabilities and means of the measured backscattering coefficients due to suspended particulate matters at the following six wavelengths: 442, 488, 532, 589, 676 and $852 \mathrm{~nm}$

把模拟的后向散射系数 $b_{\mathrm{bps}}$ 在对应波段处的平均值代人(7)式，得到 $442 、 488 、 532 、 589 、 676$ 和 $852 \mathrm{~nm}$ 处的后向散射概率 $\tilde{b}_{\mathrm{br}}$, 分别为 $0.017 、 0.017 、 0.027 、 0.033 、 0.054$ 和 0.094 , 均值为 $0.041 \pm 0.030,400-700 \mathrm{~nm}$ 范围内的均值为 $0.030 \pm 0.015$. 表明颗粒物后向散射概率不是一个常量, 与波长 $\lambda$ 存在显著的二次函数关 系(图 2), 有:

$$
\tilde{b}_{\mathrm{br}}=0.0044 \lambda^{2}-0.0163 \lambda+0.0311, R^{2}=0.9828
$$




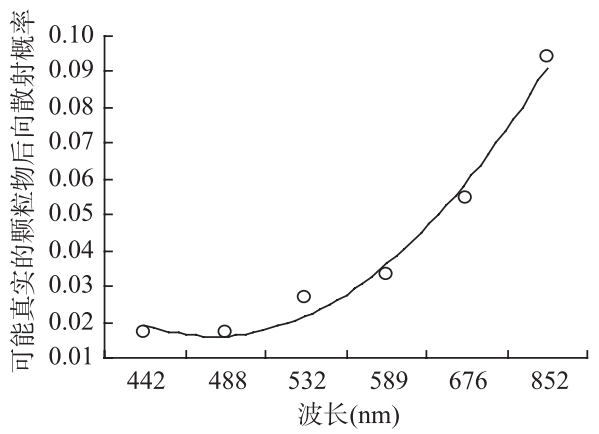

图 2 可能真实的后向散射概率与波长之间的关系 Fig.2 relationship between the possibly real backscattering probability and wavelength
使用后向散射概率 $\tilde{b}_{\mathrm{br}}$, 对 HS-6 现场测量的原始数据 重新进行校正，所得的后向散射系数(称为可能真实 的后向散射系数，即 the possibly real backscattering coefficient, $b_{\mathrm{br}}$ ) 以及模拟的后向散射系数 $b_{\mathrm{bs}}$ 在波形上 较为一致, 都随波长的不断增加而呈指数减少趋势 (图 3), 但 $b_{\mathrm{bs}}$ 明显过于理想化, $b_{\mathrm{br}}$ 较为合理. 在整个波 长范围内, 高混浊水体的 $b_{\mathrm{bs}}>b_{\mathrm{br}}$, 中混浊水体刚好相 反; 不论 $b_{\mathrm{br}}$ 还是 $b_{\mathrm{bs}}$, 都明显不同于 $b_{\mathrm{bm}}$, 在红外近红 外波段范围内发生的校正量不足的问题得以克服. 另 外，对于清澈水体(低清澈、高清澈), 从波形上看, $b_{\mathrm{br}}$ 也好于 $b_{\mathrm{bm}}$, 数量上相差较大的都发生在红外近红外 波段范围内. 上述分析，表明了 $\tilde{b}_{\mathrm{br}}$ 的合理性、适用性 和实用性.

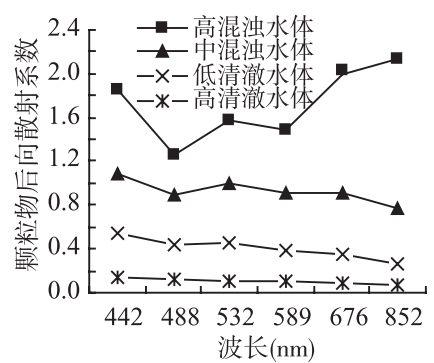

(a)

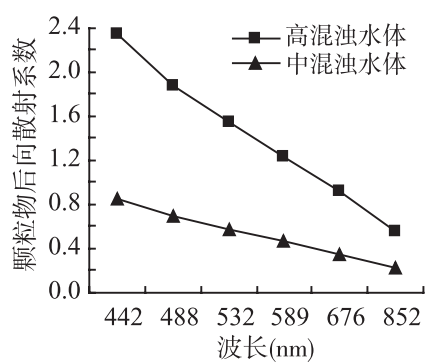

(b)

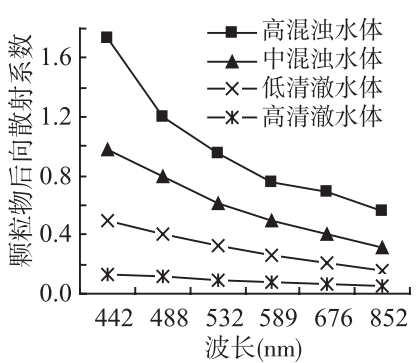

(c)

图 3 不同水体平均的颗粒物后向散射系数的对比: (a)使用 HS-6 默认的后向散射概率 0.015 校正后的 颗粒物后向散射系数; (b)模拟的后向散射系数; (c)使用本文计算的后向散射概率, 442、488、532、589、 676 和 $852 \mathrm{~nm}$ 处分别为 $0.017 、 0.017 、 0.027 、 0.033 、 0.054$ 和 0.094

Fig.3 Comparison between means of backscattering coefficient of suspended particulate matter in different waters: (a) corrected by the default backscattering probability of 0.015 in HS-6; (b) the simulated backscattering coefficient; (c) corrected by the possibly real backscattering probabilities of 0.017 at 442 $\mathrm{nm}, 0.017$ at $488 \mathrm{~nm}, 0.027$ at $532 \mathrm{~nm}, 0.033$ at $589 \mathrm{~nm}, 0.054$ at $676 \mathrm{~nm}$ and 0.094 at $852 \mathrm{~nm}$

\section{3 讨论}

后向散射系数是水色遥感半分析算法必不可少的固有光学量, 但能够非常准确地揭示目标水体的后 向散射特性比较困难. 水体的后向散射主要来自纯水和悬浮颗粒, 纯水的后向散射已经有大家公认的结 果, 但颗粒物后向散射在不同的水体中差别较大. 后向散射的现场测量过程中, 特别对于混浊水体而言, 光的迅速衰减会导致后向散射系数的低估，低估量随混浊度的增加而增加，因此准确地补偿这种由于强 衰减而引起的低估的后向散射就显得尤为重要. 本次现场实测之前, 太湖水体的光学特性还十分匮乏, 还没有确定的、先验的后向散射概率可以利用，从而给后向散射系数的现场测量和校正带来了困难. HS-6 后向散射概率的默认值(0.015)主要适用于大洋一类水体，太湖属于典型的内陆二类水体对，因此使用 0.015 作为太湖水体的后向散射概率会带来较大误差. 同后向散射相比, 吸收系数的测量相对容易, 精度 最高 ${ }^{[13]}$ ，因此通过吸收系数，使用优化算法，结合后向散射概率和颗粒物后向散射系数之间的定量关系， 可以获得较高精度的后向散射系数模拟结果, 模拟的后向散射系数更接近于真值. 许多研究把后向散射 概率看作一个定值，然而，最近的研究表明后向散射概率是一个与波长相关的量 ${ }^{[5]}$, 本文的研究证明了 这一点. 但在不同的水体中, 后向散射概率随波长的变化函数不同; Aas 等 ${ }^{[5]}$ 表明, 后向散射概率是波长 
的幂函数，可以通过 $\lambda^{-\eta}$ 来估测 ; 本文的研究表明它是波长的二次函数. 这种差别可能是由不同的颗粒属 性引起的 ${ }^{[5]}$, 但还没有得到证实. 另外，太湖后向散射概率(400-900nm 范围内均值 $0.041 \pm 0.030$ 、 400-700nm 范围内均值 0.030 0.015 )远大于海水，如 Oslo Fjord 水体 400-700nm 范围内的后向散射概率 为 $0.020 \pm 0.015^{[5]}$, 稍小于一些更混浊的水体，如 Whitlock 等 ${ }^{[4]}$ 在整个波长范围内得到的 0.059 . 本文所得 的后向散射概率是在吸收系数和遥感反射比无测量误差的假设下得出的, 是否稳定, 是否随时相而变化, 还需进一步验证. 另外，按照 Mie 理论，后向散射概率由粒子的折射指数和粒子谱共同决定. 假设水体中 粒子的矿物质构成变化不大，即折射指数相对稳定，则决定后向散射概率的应该是粒子谱分布，而粒子 谱分布又与水体浑浊度或总悬浮物浓度有关, 因此后向散射概率除了与波长相关外, 还应与悬浮颗粒浓 度有关，本文在这一方面尚未涉及.

\section{4 参考文献}

[1] Ma R, Tang J, Dai J. Bio-optical model with optimal parameter suitable for Lake Taihu in water colour remote sensing. International Journal of Remote Sensing, 2006, 27: 4303-4326.

[2] HOBI-labs Inc. Backscattering sensor calibration manual (Revision J). Available online at www.hobilabs.com (accessed 24 July 2003).

[3] Kirk JTO. Estimation of the scattering coefficient of natural waters using underwater irradiance measurements. Australian Journal of Marine and Freshwater Research, 1981, 32: 533-539.

[4] Whitlock CH, Pool LR, Usry JW et al. Comparison of reflectance with backscatter and absorption parameters for turbid waters. Applied Optics, 1981, 20: 517-522.

[5] Aas E, Høkedal J, Sørensen K. Spectral backscattering coefficient in coastal waters. International Journal of Remote Sensing, 2005, 26: 331-343.

[6] Smith RC, Baker KS. Optical properties of the clearest natural waters (200-800nm). Applied Optics, 1981, 20: 177-184.

[7] Gordon HR, Smith RC, Zaneveld JRV. Introduction to ocean optics. In: Duntley SQ ed. Ocean Optics VI. Proceedings of SPIE, 1980, 208: 1-43.

[8] Sathyendranath S, Cota G, Stuart V et al. Remote sensing of phytoplankton pigments: a comparison of empirical and theoretical approaches. International Journal of Remote Sensing, 2001, 22: 249-273.

[9] Lee Z, Carder KL, Arnone RA. Deriving inherent optical properties from water color: a multiband quasi-analytical algorithm for optically deep waters. Applied Optics, 2002, 41: 5755-5772.

[10] IOCCG. Models, parameters, and approaches that used to generate wide range of absorption and backscattering spectra. available online at www.ioccg.org/groups/lee_data.pdf. 2003 (accessed 18 May 2004).

[11] Kirk JTO. Light \& photosynthesis in aquatic ecosystems. Cambridge: Cambridge University Press, 1994.

[12] Ma R, Tang J, Dai J et al. Absorption and scattering properties of water body in Lake Taihu, China: absorption. International Journal of Remote Sensing, 2006, 27: 4275-4302.

[13] Mobley CD. Light and water: radiative transfer in natural waters. San Diego: Academic Press, 1994. 